\title{
Predisposing, Enabling, and Need Factors Associated with High Service Use in a Public Mental Health System
}

\author{
Laurie A. Lindamer $\cdot$ Lin Liu • David H. Sommerfeld • \\ David P. Folsom • William Hawthorne • Piedad Garcia • \\ Gregory A. Aarons $\cdot$ Dilip V. Jeste
}

Published online: 1 May 2011

(c) The Author(s) 2011. This article is published with open access at Springerlink.com

\begin{abstract}
The purpose of this study was twofold: (1) To investigate the individual- and system-level characteristics associated with high utilization of acute mental health services according to a widely-used theory of service useAndersen's Behavioral Model of Health Service Use -in individuals enrolled in a large, public-funded mental health system; and (2) To document service utilization by high
\end{abstract}

L. A. Lindamer $(\bowtie)$

VA Center of Excellence for Stress and Mental Health, University of California, San Diego, 3350 La Jolla Village Drive 116-A, San Diego, CA 92161, USA

e-mail: 1lindamer@ucsd.edu

L. Liu

Department of Family and Preventive Medicine, University of California, San Diego, 9500 Gilman Drive, La Jolla, CA 92093-0717, USA

D. H. Sommerfeld · W. Hawthorne - G. A. Aarons Department of Psychiatry, University of California, San Diego, 9500 Gilman Drive, La Jolla, CA 92093-0603, USA

D. P. Folsom

Departments of Psychiatry and Family Medicine, University of California, San Diego, 9500 Gilman Drive, La Jolla, CA 92093-0603, USA

P. Garcia

Behavioral Health Services, Adult/Older Adult Mental Health, Health and Human Services Agency, 3255 Camino del Rio South, San Diego, CA 92108, USA

D. V. Jeste

Departments of Psychiatry \& Neurosciences, University of California, San Diego, 9500 Gilman Drive, La Jolla, CA 92093-0603, USA use consumers prior to a transformation of the service delivery system. We analyzed data from 10,128 individuals receiving care in a large public mental health system from fiscal years 2000-2004. Subjects with information in the database for the index year (fiscal year 2000-2001) and all of the following 3 years were included in this study. Using logistic regression, we identified predisposing, enabling, and need characteristics associated with being categorized as a single-year high use consumer (HU: $>3$ acute care episodes in a single year) or multiple-year HU ( $>3$ acute care episodes in more than 1 year). Thirteen percent of the sample met the criteria for being a single-year HU and an additional $8 \%$ met the definition for multiple-year HU. Although some predisposing factors were significantly associated with an increased likelihood of being classified as a HU (younger age and female gender) relative to nonHUs, the characteristics with the strongest associations with the HU definition, when controlling for all other factors, were enabling and need factors. Homelessness was associated with $115 \%$ increase in the odds of ever being classified as a HU compared to those living independently or with family and others. Having insurance was associated with increased odds of being classified as a HU by about $19 \%$ relative to non-HUs. Attending four or more outpatient visits was an enabling factor that decreased the chances of being defined as a HU. Need factors, such as having a diagnosis of schizophrenia, bipolar disorder or other psychotic disorder or having a substance use disorder increased the likelihood of being categorized as a HU. Characteristics with the strongest association with heavy use of a public mental health system were enabling and need factors. Therefore, optimal use of public mental services may be achieved by developing and implementing interventions that address the issues of homelessness, insurance coverage, and substance use. This may be best 
achieved by the integration of mental health, intensive case management, and supportive housing, as well as other social services.

Keywords Mental health - Service utilization · System redesign

\section{Introduction}

The disproportionate use of services by a subset of mental health service users, described here as "high use consumers" (HU), has been demonstrated in large, cross-sectional (Sullivan et al. 1993; Dhossche and Ghani 1998; Pasic et al. 2005; Hansen and Elliott 1993), longitudinal, (Saarento et al. 1998; Segal et al. 1998) and case control studies (Arfken et al. 2004; Silva et al. 2009) of emergency psychiatric care in several service systems in community and veterans samples (Hansen and Elliott 1993). Although the definitions of high utilization differ among studies, between 5 and $18 \%$ of mental health services users account for utilization of $27-63 \%$ of the services (Sullivan et al. 1993; Hansen and Elliott 1993; Dhossche and Ghani 1998; Saarento et al. 1998).

Disproportionately high use of mental health services by a small proportion of consumers is an issue that has challenged service delivery systems for decades. Several studies have investigated the sociodemographic and clinical variables associated with high service utilization in attempts to identify common characteristics that might inform the development of interventions to improve both delivery and quality of care (Surles and McGurrin 1987; Surber et al. 1987; Quinlivan et al. 1995; Sullivan et al. 1993; Witheridge et al. 1982). Few studies, however, have employed a theoretical framework to understand factors related to mental health service use, which could be helpful in designing program or system level interventions (Dhingra et al. 2010). According to a widely-used theory of service use, Andersen's Behavioral Model of Health Service Use (Andersen and Newman 1973; Andersen 1995), the characteristics related to service use include more than need for services. Andersen and colleagues' theory proposed that factors associated with health service use can be grouped into three categories: predisposing (characteristics of the individual, i.e., age, gender, race), enabling (system or structural factors, i.e., living arrangement and insurance status), and need (severity of illness) factors. Predisposing factors associated with high service utilization include male gender (Sullivan et al. 1993), younger age (Sullivan et al. 1993; Silva et al. 2009; Surles and McGurrin 1987), and non-Caucasian ethnicity (Sullivan et al. 1993; Havassy and Hopkin 1989). Enabling factors related to high utilization consist of single marital status (Sullivan et al. 1993), unemployment (Sullivan et al. 1993; Dhossche and Ghani 1998; Havassy and Hopkin 1989), homelessness (Dhossche and Ghani 1998; Pasic et al. 2005; Arfken et al. 2004), enrollment in a mental health plan (Pasic et al. 2005; Segal et al. 1998), and a less favorable pattern of service use (i.e., more hospitalizations and/or fewer outpatient visits) (Hansen and Elliott 1993; Pasic et al. 2005; Arfken et al. 2004; Silva et al. 2009). Need factors, such as a diagnosis of schizophrenia or other psychotic disorder (Sullivan et al. 1993; Dhossche and Ghani 1998; Hansen and Elliott 1993; Segal et al. 1998; Silva et al. 2009; Surles and McGurrin 1987), have consistently been shown to be associated with increase service utilization; whereas substance use has been found to increase utilization in some cases (Pasic et al. 2005; Surber et al. 1987), but not in others (Arfken et al. 2004). There is some suggestion in the literature that factors related to high service use may vary depending on chronicity (Pasic et al. 2005), i.e., high use of services for a single year versus several years. Knowledge of predisposing, enabling, and need characteristics associated with patterns of high service use, for a year or multiple years may inform mental health systems about the nature of the interventions that would be needed to optimize care for both the individual and the system.

Many interventions to address some combination of predisposing, enabling, and need factors have been developed to optimize service use for individuals who have relied heavily on acute and intensive services. These include Assertive Community Treatment, Intensive Case Management, and integrated care for those dually diagnosed with a psychiatric illness and substance abuse (Drake et al. 2001). Most of these interventions have focused on team-based, integrated approaches to address the multiple medical, psychiatric, and social issues common to the high use consumers. The more effective treatments have targeted all of the Andersen domains, but these interventions are few and resource intensive. Identifying important individual and system characteristics that are associated with high mental health service use is an essential step if effective and cost effective interventions are to be developed and implemented.

Not only is mental health service use influenced by characteristics of the individual, but also it is influenced by the structure of the service delivery system (Snowden 2007). In California, the mental health system has been undergoing substantial changes due to the enactment of the 2004 Mental Health Services Act (MHSA) (Cashin et al. 2008). This act provided resources to support the transformation of the mental health service system into one that is more recovery focused, reaches out to underserved and un-served populations, promotes innovation, and integrates services across multiple areas (e.g., health, housing, social assistance) if needed. These system transformation activities have the potential to influence the size and composition of the high use consumer population by altering the types of services available to existing mental health consumers and 
identifying new consumers who could benefit from mental health services. Given that the system transformation is currently in process, this paper utilizes data prior to the implementation of the MHSA, which corresponds to a more traditional service delivery system. These data provide a baseline from which to compare the influence of the MHSA system transformation on the high use consumer population following more extensive implementation and operation of the MHSA services in the coming years. Although the MHSA is specific to California, many local and regional mental health systems are considering transformation of service delivery so that information gleaned from changes in the San Diego County may guide the administrators and policy makers of other systems.

In summary, many studies have investigated the sociodemographic and clinical characteristics associated with access to mental health services, particularly emergency services. However, there is considerable methodological variation among the studies, including differences in the sample and study design, length of observation, definitions of high utilization, and types of services included. Inconsistency might also be furthered by different consumer subgroups based on use (Pasic et al. 2005). Finally, few studies have employed a theoretical framework to understand factors related to service use, which may assist in the identification of potential interventions to improve service use and quality of care. The purpose of the present study was to investigate the use of acute mental health services beyond emergency department use in a large sample of public mental health users on whom we had 4 years of data. We used a definition of HU that was consistent with previous reports of service utilization, and we examined use by subgroups of $\mathrm{HU}$, specifically those using more than three acute services in 1 year or more than 1 year. These data provide a baseline of the size and composition of the high use consumer population prior the MHSA induced service transformations. We hypothesized that: (1) There is a small group of enrollees that use disproportionately more acute mental health services; and (2) Use of such services is related to predisposing factors of male gender, younger age, enabling factors of homelessness and outpatient service use, and need related factors, such as a diagnosis of schizophrenia and substance use; (3) Enabling and need factors differ between one time HUs and multiple-year HUs with multiple HUs having more barriers to service use and greater need.

\section{Methods}

\section{Data Source}

We analyzed data from the public mental health services database of the San Diego County Adult and Older Adult
Mental Health Services (AOAMHS) from fiscal years 2000-2004. In California, public mental health services are organized by counties that provide mental healthcare either directly or through independent subcontractors. Data on client characteristics and service use of individuals enrolled in any service delivered by San Diego County or its contracted providers are maintained in a Management Information System (MIS) that is managed by a third party contractor. As part of an ongoing collaboration between our NIMH-funded Advanced Center for Innovation in Services and Intervention Research and AOAMHS, we have extensive experience with managing and analyzing this database (Folsom et al. 2006; Jin et al. 2003; Folsom et al. 2005). Data originating from files containing multiple episodes of care are aggregated at the client level into a single data analysis file that includes indicators of sociodemographic and clinical characteristics as well as service utilization records. Use of these MIS data for this secondary analysis was approved by the UCSD Institutional Review Board and the San Diego County Research Committee.

\section{Sample}

At the time data collection began, San Diego County had a population of roughly 2.8 million people, making it the second largest county in the state of California and ranking 16th in population of all metropolitan areas in the U.S. The county encompasses urban, suburban, semi-rural, and rural communities and has an ethnically diverse population, with 55\% non-Latino Whites, 27\% Latinos, 9\% Asian Americans, and 5\% African-Americans (U.S.Census Bureau and SANDAG 2000).

Adult subjects with information in the database for the index year (fiscal year 2000-2001) and all of the following 3 years were included in the study. Persons were excluded if there was no recorded diagnosis, no information about any service use in fiscal year 2000-2001, missing information regarding their use of acute mental health services (emergency psychiatric unit (EPU), psychiatric emergency response team (PERT), inpatient psychiatric hospital, and crisis residential during any of these four years or missing selected baseline characteristics. Among 15,323 subjects on whom there were data for four fiscal years, 10,128 subjects had sufficient data to be included in the final sample for the analyses. This group, therefore, had used services in the public mental health system at least once in all 4 years.

Predisposing, Enabling, and Need Factors

Information on age, gender, ethnicity, living situation, insurance coverage, and presence of a co-morbid substance use disorder was derived from the San Diego County MIS database for AOAMHS for the index year 2000-2001. 


\section{Predisposing Factors}

Age was grouped into two categories ( $<45$ and $\geq 45$ years) for the analysis. On the basis of self-identification, ethnicity was classified as Caucasian, African-American, Latino, Asian-American, Native-American or Other. To ensure sufficient race/ethnic group sample size for the analyses, Asian-American, Native-American, and Other were collapsed into a single "other" category.

\section{Enabling Factors}

Living situation was recorded at admission to and discharge from each type of service and coded into the following categories: living independently or with family or others, residing in a board-and-care (assisted living) facility, being incarcerated, being homeless, or other. We used the modal living situation for the index year 2000-2001 (defined as the most frequent living situation recorded for the year) to define residential status. Medicaid insurance coverage was coded as a dichotomous variable (no/yes) based on the status recorded in fiscal year 2000-2001. As appropriate use of mental health services includes access to and use of outpatient care, we compared the number of outpatient visits according to the following three categories: three or fewer, four to six, and greater than six visits per year. It could be argued that outpatient visits could be a representation of need for services rather than an enabling factor influencing the use of services. We chose to categorize outpatient visits as an enabling factor reasoning that more outpatient care may be associated with less utilization of acute services, a pattern of service use that is usually more optimal for both the individual and the system than excessive use of costly acute services.

\section{Need Factors}

Substance use disorder diagnoses were categorized as the presence or absence of any substance use diagnosis assigned in any care setting for the fiscal year 2000-2001. Individuals were classified into five diagnostic groups based on the diagnosis recorded in the index year: schizophrenia, bipolar disorder, major depressive disorder, other psychotic disorders, and other. If more than one diagnosis was recorded, we assigned diagnostic categories based on a hierarchical algorithm used in previous studies in which the most severe diagnosis given (i.e., schizophrenia, bipolar disorder, major depressive disorder, other psychotic disorder) (Folsom et al. 2005; Jin et al. 2003) was used. The use of this algorithm is supported by our previous finding that the diagnosis of schizophrenia is more reliable than a diagnosis of depression in this database (Folsom et al. 2006).
Service Utilization

Using the AOAMHS data, we categorized mental health services into five groups: emergency psychiatric unit (EPU), psychiatric emergency response team (PERT), inpatient psychiatric hospital, crisis residential, and outpatient. Each client was considered to have received service in a particular category if at least one billable encounter in that service category for the fiscal year 2000-2001 was recorded. For each service group, a variable was created by aggregating episodic visits for each client into a dichotomous presence or absence of the service category. EPU treatment included service in the AOAMHS-operated psychiatric emergency unit, but did not include emergency psychiatric treatment in any of the other emergency departments in the county. We also included visits made by the Psychiatric Emergency Response Team (PERT), which consists of police officers and mental health clinicians who respond to 911 calls involving persons with psychiatric disorders. Inpatient psychiatric hospitalization included admission to 11 feefor-service psychiatric hospital programs providing acute inpatient care under contract with AOAMHS or admission to the San Diego County Psychiatric Hospital. Crisis residential treatment included admission to any of a network of six crisis residential programs regionally located throughout San Diego County. These programs provide short-term acute residential treatment services as an alternative to voluntary hospitalization. Three types of outpatient visits were considered: appointments for medication management, psychotherapy, and case management. Outpatient use was categorized into three groups: up to three outpatient visits, four to six outpatient visits, and more than six outpatient visits base on the assumption that the minimum standard of care is quarterly medication management visits. Only inpatient, EPU, PERT and crisis residential visits were used for categorizing HUs.

\section{Definition of High Use Consumers}

Subjects were defined as HUs if they used inpatient, EPU, PERT, or crisis residential services three or more times in a fiscal year. We chose this definition of HU for the following reasons: (1) It was consistent with other definitions in the literature (Sullivan et al. 1993; Silva et al. 2009; Pasic et al. 2005; Hansen and Elliott 1993); and (2) The median number of acute service episodes per year of the multiple-year HU was two. To further verify the use of three acute visits for the definition of HU, we conducted a sensitivity analysis using a cut-off of four visits and found similar results as with a cut-off of three but with inadequate samples sizes per cell for some analyses. We categorized the individuals into three groups: those who were never 
classified as HUs in any of the 4 years, those were considered a HUs for one of the 4 years (single-year HU), and those who met the definition of HUs for more than one of the 4 years (multiple-year HU).

\section{Statistical Analyses}

Chi-square tests were used to study differences in categorical baseline predisposing, enabling, and need characteristics among the three mental health service utilization groups, and Kruskal-Wallis tests were used to compare the number of episodes of EPU, inpatient, crisis residential and PERT among the three mental health service utilization groups in the index year. Pairwise comparisons with Hochberg adjustment for $p$-values were performed for all significant tests. We next conducted two sets of analyses. First, we utilized logistic regression to compare the associations between baseline predisposing, enabling, and need factors and ever being a HU (comprised of one-time HU and multiple-year HU). The logistic regression can be expressed as $\log \left(p_{1} / p_{0}\right)=\beta_{0}+\beta_{1} x_{1}+\cdots+\beta_{p} x_{p}$, where $p_{1}$ is the probability that the subject is in one-time or multiple-year $\mathrm{HU}$ group, $p_{0}$ is the probability that the subject has never been a HU and $x_{\mathrm{i}}$ 's are independent variables or predictors. Second, we used multinomial logistic regression to study the association between the three mental health service groups (never being a HU, one-time HU and multiple-year HU) and baseline predisposing, enabling, and need factors. The multinomial logistic regression can be expressed as $\log \left(p_{i} / p_{0}\right)=\beta_{0}+\beta_{1} x_{1}+\cdots+\beta_{p} x_{p}$, where $p_{\mathrm{i}}$ is the probability that the subject is in ith group, $p_{0}$ is the probability that the subject is in the reference group. In this analysis, the group 'never being a HU' was specified as a reference group. To compare the probability of being a multiple HU and the probability of being a single-year HU, we also ran the multinomial logistic regression with 'single-year HU' specified as a reference category. Influential observations were assessed using Cook's statistics and leverages. Since excluding the influential observations did not change the results significantly, the analysis results with all observations included were reported. A two-tailed $p$-value $<0.05$ indicated statistical significance. Statistical analyses were performed using the open source statistical package (version 2.81; R Development Core Team 2008).

\section{Results}

\section{Sample Characteristics}

In this large public mental health system, about $57 \%$ of the subjects were age 45 years or younger, slightly more than half $(54 \%)$ were female, $58 \%$ were Caucasian, and most of them $(68 \%)$ were living independently with families or with others. Forty-two percent of subjects were diagnosed with schizophrenia, $24 \%$ with depression, $12 \%$ with bipolar disorder, $5 \%$ with other psychotic disorders, and $17 \%$ with other psychiatric diagnoses. A concomitant substance use disorder was reported in $24 \%$ of the total sample. Nearly $60 \%$ had Medicaid insurance, and most subjects (72\%) had more than 6 outpatient visits.

A large majority (79\%) of the 10,128 persons receiving care from the county mental health system did not meet our criteria for being classified as a HU; whereas $13 \%$ met the criteria for being an HU in 1 year and an additional $8 \%$ were classified as HUs in more than one of the 4 years (multipleyear HUs) (Table 1). The use of acute or emergency care services varied among the groups. As expected, more episodes of EPU, inpatient, crisis residential, and PERT were recorded for the HU groups in the index year (Table 2).

Because the results from multinomial regression analyses of the non-HU, one-time $\mathrm{HU}$, and multiple-year $\mathrm{HU}$ differed almost exclusively in magnitude and not in pattern from the results of logistic regression analysis of the non$\mathrm{HU}$ and ever HU (data available upon request), we report only the results of the logistic regression analyses that assess the relationship of the baseline characteristics and the likelihood of ever being in the HU group (includes single-year HU and multiple-year HU). We have, however, presented descriptive information for all groups in Table 1. All baseline predisposing, enabling, and need factors shown in Table 1 were included in the logistic regression model to study the association between these characteristics and mental health service use.

Estimated odds ratios and $p$-values from logistic regressions are presented in Table 3. Adjusted for other variables in the model, the predisposing factors of younger age $(p<0.001, \mathrm{OR}=1.91)$ and female gender $(p=0.004$, $\mathrm{OR}=1.17$ ) were associated with an increased odds of being classified as a HU. When we used age as a continuous variable, the results were similar. The race and gender interaction was not statistically significant in any of the models (i.e., with age as a continuous or categorical variable). Ethnicity was also related to service use: the likelihood of being a HU was lower for the Latino $(p=0.013$, $\mathrm{OR}=0.84)$ and "other" $(p=0.012, \mathrm{OR}=0.77)$ race/ ethnicity group compared to Caucasians.

Also as shown in Table 3 examining enabling factors, we found that persons living in a residential or justice-related facility and having 4-6 or more than 6 outpatient visits (all $p<0.0001)$ were significantly less likely to be classified as HU. Persons who were homeless $(p<0.0001, \mathrm{OR}=2.15)$ and those having Medicaid insurance $(p<0.0001, \mathrm{OR}=$ 1.19) were significantly more likely to be a HU.

Table 3 also shows that need factors were also significantly associated with HU classification. Individuals with a 
Table 1 Predisposing, enabling, and need characteristics (percentages) for overall and groups of public mental health service utilizers

\begin{tabular}{|c|c|c|c|c|c|}
\hline & $\begin{array}{l}\text { Overall } \\
\mathrm{N}=10,128\end{array}$ & $\begin{array}{l}\text { Never high use } \\
\text { consumers } \\
N=7,977\end{array}$ & $\begin{array}{l}\text { Single-year high use } \\
\text { consumers } \\
\mathrm{N}=1,310\end{array}$ & $\begin{array}{l}\text { Multiple-year high use } \\
\text { consumers } \\
\mathrm{N}=841\end{array}$ & $\begin{array}{l}\text { Ever high use } \\
\text { consumers }^{\mathrm{a}} \\
\mathrm{N}=2,151\end{array}$ \\
\hline \multicolumn{6}{|l|}{ Predisposing } \\
\hline \multicolumn{6}{|l|}{ Age } \\
\hline$\geq 45$ years & 43 & 47 & 30 & 25 & 28 \\
\hline$<45$ years & 57 & 53 & 70 & 75 & 72 \\
\hline \multicolumn{6}{|l|}{ Gender } \\
\hline Male & 46 & 45 & 51 & 52 & 52 \\
\hline Female & 54 & 55 & 49 & 48 & 48 \\
\hline \multicolumn{6}{|l|}{ Ethnicity } \\
\hline Caucasian & 58 & 58 & 58 & 61 & 59 \\
\hline Latino & 20 & 20 & 18 & 17 & 17 \\
\hline African American & 13 & 12 & 17 & 16 & 17 \\
\hline Other & 9 & 10 & 7 & 7 & 7 \\
\hline \multicolumn{6}{|l|}{ Living situation } \\
\hline Independent/Family/Others & 68 & 69 & 65 & 61 & 63 \\
\hline Residential & 11 & 11 & 9 & 8 & 9 \\
\hline Justice & 9 & 8 & 11 & 10 & 11 \\
\hline Homeless & 6 & 4 & 9 & 16 & 12 \\
\hline Other & 7 & 7 & 6 & 5 & 6 \\
\hline \multicolumn{6}{|l|}{ Enabling } \\
\hline \multicolumn{6}{|l|}{ Medicaid insurance } \\
\hline No & 41 & 41 & 42 & 33 & 38 \\
\hline Yes & 59 & 59 & 58 & 67 & 62 \\
\hline \multicolumn{6}{|l|}{ Outpatient service use } \\
\hline$\leq 3$ visits & 20 & 18 & 26 & 28 & 27 \\
\hline $4-6$ visits & 8 & 9 & 7 & 6 & 7 \\
\hline$>6$ visits & 72 & 73 & 67 & 66 & 66 \\
\hline \multicolumn{6}{|l|}{ Need } \\
\hline \multicolumn{6}{|l|}{ Psych diagnosis } \\
\hline Depression & 24 & 26 & 17 & 9 & 14 \\
\hline Schizophrenia & 42 & 37 & 54 & 67 & 59 \\
\hline Bipolar & 12 & 12 & 12 & 14 & 13 \\
\hline Other psychosis & 5 & 5 & 7 & 5 & 6 \\
\hline Other & 17 & 20 & 11 & 5 & 8 \\
\hline \multicolumn{6}{|l|}{ Substance use disorder } \\
\hline Yes & 24 & 19 & 38 & 51 & 43 \\
\hline No & 76 & 81 & 62 & 49 & 57 \\
\hline
\end{tabular}

${ }^{a}$ Combines single-year and multiple-year high use consumers

schizophrenia diagnosis had odds of being categorized an HU that were three times the odds for those with depression. Bipolar and other psychotic disorder diagnoses increased the odds of being a $\mathrm{HU}$ by $70 \%$ relative to a depression diagnosis. Subjects with a concomitant substance use disorder had three times the odds of being categorized as a HU compared to those without a concomitant substance use disorder.

\section{Discussion}

The majority of individuals receiving mental health services from a public system were not classified as $\mathrm{HU}$, that is, as having used three or more acute (inpatient, crisis residential, EPU, or PERT) services in a year. About $20 \%$ of enrollees were classified as a HU in one or more years. The factors most strongly associated with being a HU 
Table 2 Mean (standard deviations) of number of visits to acute mental health services in the index year for groups of service utilizers

\begin{tabular}{lllr}
\hline & Never high use consumers & Single-year high use consumers & $\begin{array}{l}\text { Multiple-year high use consumers } \\
\mathrm{N}=841\end{array}$ \\
\hline EPU & $\mathrm{N}=7,977$ & $1.07(1.52)$ & $1.8(2.44)$ \\
PERT & $0.13(0.39)$ & $0.22(0.61)$ & $0.45(1.03)$ \\
Inpatient & $0.02(0.17)$ & $0.55(0.97)$ & $1.31(1.80)$ \\
Crisis residential & $0.05(0.24)$ & $0.23(0.58)$ & $0.64(1.17)$ \\
\hline
\end{tabular}

$E P U$ Emergency psychiatric unit, PERT psychiatric emergency response team

All comparisons are significant with $p$-value $<0.0001$

versus a non-HU, controlling for other variables, were enabling factors of homelessness, medical insurance, and minimal use of outpatient services, as well as need factors, including a substance use disorder and a diagnosis of schizophrenia, bipolar or other psychotic disorder. Factors from all categories of the Andersen model-predisposing, enabling, and need-were significantly associated with a higher probability of ever having been classified as HU: younger age, female gender, homelessness, substance use disorder, medical insurance, and diagnosis of schizophrenia, bipolar disorder or other psychotic disorder. Several factors were significantly related to a lower likelihood of being a HU. These included Latino or "other" race, living in a board and care home or jail, having four or more outpatient visits per year, and having a diagnosis of other psychiatric disorder.

In the 1990s, Quinliven and others reported on the characteristics of high utilizers in San Diego County (Quinlivan et al. 1995; Quinlivan and McWhirter 1996). Using a definition of high utilization as three or more visits to the emergency department, they found that the majority of this group had the diagnosis of schizophrenia or schizoaffective disorder $(68 \%)$, were more slightly more likely to be women $(56 \%)$, were mostly Caucasian (57\%) and were mostly young ( $88 \%$ were age 50 or less). Our sample had a higher proportion of persons over the age of 45 years but were otherwise similar to the group identified by Quinliven and colleagues in the early 1990s suggesting that the profile of a high use consumer has varied little over the decades.

Consistent with most previous reports in the literature, we found that a small proportion of individuals in the public mental health system were identified as high use consumers (Sullivan et al. 1993; Dhossche and Ghani 1998; Hansen and Elliott 1993; Saarento et al. 1998; Segal et al. 1998; Chaput and Lebel 2007). This finding is robust in that it is seen across many studies that examine different service systems and use different definitions of high service utilization. Therefore, identifying characteristics of such a group or groups of individuals is crucial to the development and implementation of solutions to optimize service use for the benefit of both the consumer and the service delivery system.

Many of the predisposing characteristics found in other studies to be associated with high mental health service utilization were observed to have significant relationships with being categorized as a HU in our study. As we predicted, and as others have reported (Sullivan et al. 1993; Silva et al. 2009), we found that younger age was associated with a higher likelihood of being classified as an ever HU when controlling for all other predisposing, enabling, and need factors. Female gender was significantly associated with an increased likelihood of being classified as an ever HU. Most previous research has failed to find a gender difference in service utilization (Arfken et al. 2004; Pasic et al. 2005; Chaput and Lebel 2007) or reported that male gender was related to high utilization (Sullivan et al. 1993; Young et al. 2005; Saarento et al. 1998).Our finding that female gender was associated with higher likelihood of being defined as a HU may reflect gender differences in treatment seeking. Other literature has reported that women are more likely to use mental health services (Mackenzie et al. 2006), but the relationship between gender and high utilization was not investigated. Alternatively, there could be gender differences in the systems for which men and women receive care. For example, men who are high use consumers of mental health services due to the severity of illness may be more likely to get services in the justice system if they exhibit dangerous behaviors toward others and are subsequently incarcerated.

Race/ethnicity has been found to be predictor of high service utilization in some (Sullivan et al. 1993; Young et al. 2005), but not all (Segal et al. 1998; Pasic et al. 2005) studies. In this study, we found that Latino race was associated with a decreased likelihood of being classified as an HU as was the "other" category that included AsianAmerican, Native-American, and other race. Other studies have also found ethnic/cultural differences in the use of mental health services (Neighbors et al. 2007). Unwillingness to seek care and/or willingness to provide care in the home that is observed in minority families may extend to the use of acute psychiatric services (Snowden 2007). 
Table 3 Associations between mental health service utilization and predisposing, enabling, and need characteristics using logistic regression

\begin{tabular}{|c|c|c|}
\hline & \multicolumn{2}{|c|}{ Ever HU vs. never } \\
\hline & Odds ratio & $p$-value \\
\hline \multicolumn{3}{|l|}{ Age } \\
\hline$\geq 45$ years (reference) & - & - \\
\hline$<45$ years & $1.91(1.71,2.14)$ & $<0.0001$ \\
\hline \multicolumn{3}{|l|}{ Gender } \\
\hline Male & - & - \\
\hline Female & $1.17(1.05,1.31)$ & 0.004 \\
\hline \multicolumn{3}{|l|}{ Ethnicity } \\
\hline Caucasian (reference) & - & - \\
\hline Latino & $0.84(0.73,0.96)$ & 0.013 \\
\hline African American & $1.08(0.93,1.26)$ & 0.327 \\
\hline Other & $0.77(0.63,0.94)$ & 0.012 \\
\hline \multicolumn{3}{|l|}{ Living situation } \\
\hline $\begin{array}{l}\text { Independent/Family/Others } \\
\text { (reference) }\end{array}$ & - & - \\
\hline Residential & $0.58(0.48,0.70)$ & $<0.0001$ \\
\hline Justice & $0.61(0.50,0.74)$ & $<0.0001$ \\
\hline Homeless & $2.15(1.77,2.62)$ & $<0.0001$ \\
\hline Other & $0.76(0.61,0.94)$ & 0.012 \\
\hline \multicolumn{3}{|l|}{ Psychiatric diagnosis } \\
\hline Depression (reference) & - & \\
\hline Schizophrenia & $3.32(2.85,3.89)$ & $<0.0001$ \\
\hline Bipolar & $1.71(1.41,2.08)$ & $<0.0001$ \\
\hline Other psychosis & $1.71(1.34,2.20)$ & $<0.0001$ \\
\hline Other & $0.66(0.53,0.81)$ & 0.0001 \\
\hline \multicolumn{3}{|l|}{ Substance use disorder } \\
\hline No (reference) & - & \\
\hline Yes & $3.13(2.79,3.52)$ & $<0.0001$ \\
\hline \multicolumn{3}{|l|}{ Medi-Cal insurance } \\
\hline No (reference) & - & \\
\hline Yes & $1.19(1.05,1.35)$ & 0.006 \\
\hline \multicolumn{3}{|l|}{ Outpatient service use } \\
\hline$\leq 3$ visits (reference) & - & $<0.0001$ \\
\hline $4-6$ visits & $0.49(0.39,0.61)$ & $<0.0001$ \\
\hline$>6$ visits & $0.52(0.45,0.60)$ & \\
\hline
\end{tabular}

Our finding that the enabling factor, homelessness, is associated with high use of mental health services has been noted by others (Arfken et al. 2004; Pasic et al. 2005). In our study, those residing in residential settings and jail were less likely than those living independently to be classified as HU. In contrast, one other study (Pasic et al. 2005) noted that a history of incarceration was related to increased use of mental health services perhaps due the disruption of continuity of care. In regard to those who had some residential services, our results suggest that interventions that include assistance with making and attending appointments and taking medication may lead to decreased use of mental health services. Some studies (Martinez and Burt 2006) have found that permanent supportive housing reduced emergency room use and episodes of inpatient hospitalizations in a group of individuals with comorbid psychiatric and substance use disorders. Gilmer and colleagues (Gilmer et al. 2003) also found significant reductions in the use of hospitalization and other emergency services for adults with serious mental disorders enrolled in a supported housing and assertive community treatment program compared to a matched control group not enrolled in the program. Consistent with the implications of these findings, San Diego County has prioritized supportive housing and developed many new housing resources using funds from the recent enactment of the MHSA.

The association of being enrolled in a medical plan and high utilization of services has been previously reported in the literature (Pasic et al. 2005; Segal et al. 1998). We found that the odds of being a HU was increased by $19 \%$ for Medicaid beneficiaries compared to those without Medicaid. Since we determined Medicaid status from the index year, the association between insurance coverage and use of services is most likely due to the fact that individuals with insurance (predominantly Medicaid) were more likely to be hospitalized over the course of the study period. This finding is consistent with a study of enrollment status in a public managed mental health care plan and emergency psychiatric service use that reported that clients with current or previous Medicaid enrollment had more lifetime inpatient hospitalizations than those never enrolled (Wingerson et al. 2001).

As predicted, we found that need was related to mental health service use. Our findings that the likelihood of being classified as a HU increased with higher need for services (i.e., diagnosis of schizophrenia, bipolar disorder, and other psychotic disorder and co-morbid substance use) are consistent with other studies in the literature (Sullivan et al. 1993; Dhossche and Ghani 1998; Hansen and Elliott 1993; Segal et al. 1998; Silva et al. 2009). Some (Pasic et al. 2005) all studies (Arfken et al. 2004) have found a relationship between substance use disorder diagnosis and high utilization of services.

In contrast to many previous studies, we examined acute mental health services beyond just emergency department use, including EPU, PERT, inpatient, and crisis residential services. However, a significant limitation of this study is that we did not differentiate between a HU of services who used multiple acute services in one episode of illness (e.g., presented at EPU, was hospitalized, then discharged to a crisis residential facility) and a person who used three services over more than one episode of illness. The 
characteristics associated with these patterns of use may differ. Another limitation includes the lack of length of service and cost data, which could yield important information about patterns of service utilization. Furthermore, the secondary use of an administrative database limited the number and types of factors (enabling and need) that we could investigate. In addition, the MIS dataset used in the analyses did not include emergency mental health services provided at private fee for service hospital emergency departments which provide services to Medicaid beneficiaries. Finally, to the extent that the types of acute psychiatric differ from those offered in San Diego County, generalizability may be limited.

Despite these limitations, there were several strengths of our study. We analyzed four consecutive years of data from a large public mental health system which allowed comparison of one-time HUs and multiple-year HUs across time. We also included a greater range of emergency service use across the 4 year study period which allowed inclusion of more individuals in the analysis.

In summary, many of the enabling and need factors found to be associated with the likelihood of being a high use consumer of mental health services (e.g., substance abuse, homelessness, insurance coverage, and minimal outpatient service use) suggest the importance of recognizing the relationship of the public mental health system to other public and private sector service systems. In this regard addressing the needs of HUs of mental health services will likely require the involvement of partnerships across service sectors. Therefore, optimal use of public mental services might be achieved by developing and implementing interventions that also attend to other concerns such as homelessness, insurance coverage, and substance use.

This finding is consistent with one of the major service initiatives instituted through the MHSA-full service partnerships (FSP). The FSP programs are explicitly designed to link persons with mental health concerns to a range of other needed services with the goal of bringing about long-term improvement in mental health status and overall functioning in many facets of life. As the result of the implementation of these programs, we hope to see reductions in the overall numbers of persons characterized as HUs of acute mental health services and the potential for changes in the characteristics associated with HUs.

Acknowledgment This work was supported, in part, by the National Institute of Mental Health grants MH080002, MH19934, MH71539 and the VA Center of Excellence for Stress and Mental Health.

Open Access This article is distributed under the terms of the Creative Commons Attribution Noncommercial License which permits any noncommercial use, distribution, and reproduction in any medium, provided the original author(s) and source are credited.

\section{References}

R Development Core Team. (2008). R: A language and environment for statistical computing. Vienna, Austria: R Foundation for Statistical Computing. ISBN 3-900051-07-0. http://www.Rproject.org.

Andersen, R. M. (1995). Revisiting the behavioral model and access to medical care: Does it matter? Journal of Health and Social Behavior, 36, 1-10.

Andersen, R., \& Newman, J. F. (1973). Societal and individual determinants of medical care utilization in the United States. Milbank Memorial Fund Quarterly, 51, 95-124.

Arfken, C. L., Zeman, L. L., Yeager, L., White, A., Mischel, E., \& Amirsadri, A. (2004). Case-control study of frequent visitors to an urban psychiatric emergency service. Psychiatric Services, 55, 295-301.

Cashin, C., Scheffler, R., Felton, M., Adams, N., \& Miller, L. (2008). Transformation of the California mental health system: Stakeholder-driven planning as a transformational activity. Psychiatric Services, 59, 1107-1114.

Chaput, Y. J., \& Lebel, M. J. (2007). Demographic and clinical profiles of patients who make multiple visits to psychiatric emergency services. Psychiatric Services, 58, 335-341.

Dhingra, S. S., Zack, M., Strine, T., Pearson, W. S., \& Balluz, L. (2010). Determining prevalence and correlates of psychiatric treatment with Andersen's behavioral model of health services use. Psychiatric Service, 61, 524-528.

Dhossche, D. M., \& Ghani, S. O. (1998). A study on recidivism in the psychiatric emergency room. Annals of Clinical Psychiatry, 10, 59-67.

Drake, R. E., Essock, S. M., \& Shaner, A. (2001). Implementing dual diagnosis services for clients with severe mental illness. Psychiatric Services, 52, 469-476.

Folsom, D. P., Hawthorne, W., Lindamer, L., Gilmer, T., Bailey, A., Golshan, S., et al. (2005). Prevalence and risk factors for homelessness and utilization of mental health services among 10,340 patients with serious mental illness in a large public mental health system. American Journal of Psychiatry, 162, 370-376.

Folsom, D. P., Lindamer, L., Montross, L., Hawthorne, W., Golshan, S., Hough, R., et al. (2006). Diagnostic variability for schizophrenia and major depression in a large public mental healthcare system district. Psychiatry Research, 144, 167-175.

Gilmer, T. P., Folsom, D. P., Hawthorne, W., Lindamer, L. A., Hough, R. L., Garcia, P., et al. (2003). Assisted living and use of mental health services among Medicaid beneficiaries with schizophrenia. Journal of Mental Health Economics and Policy, 6, 59-65.

Hansen, T. E., \& Elliott, K. D. (1993). Frequent psychiatric visitors to a Veterans Affairs medical center emergency care unit. Hospital \& Community Psychiatry, 44, 372-375.

Havassy, B. E., \& Hopkin, J. T. (1989). Factors predicting utilization of acute psychiatric inpatient services by frequently hospitalized patients. Hospital \& Community Psychiatry, 40, 820-823.

Jin, H., Folsom, D. P., Lindamer, L., Bailey, A., Hawthorne, W., Garcia, P., et al. (2003). Patterns of service use by age in patients with schizophrenia in a large public mental health system. American Journal of Geriatric Psychiatry, 11, 525-533.

Mackenzie, C. S., Gekoski, W. L., \& Knox, F. J. (2006). Age, gender, and the underutilization of mental health services: The influence of help-seeking attitudes. Aging and Mental Health, 10, 574-582.

Martinez, T. E., \& Burt, M. R. (2006). Impact of permanent supportive housing on the use of acute care health services by homeless adults. Psychiatric Services, 57, 992-999. 
Neighbors, H. W., Caldwell, C., Williams, D. R., Nesse, R., Taylor, R. J., Bullard, K. M., et al. (2007). Race, ethnicity, and the use of services for mental disorders: Results from the national survey of American life. Archives of General Psychiatry, 64, 485-494.

Pasic, J., Russo, J., \& Roy-Byrne, P. (2005). High utilizers of psychiatric emergency services. Psychiatric Services, 56, 678684.

Quinlivan, R., Hough, R. L., Cowell, A., Beach, C., Hofstetter, R., \& Kenworthy, K. (1995). Service utilization and costs of care for severely mentally ill clients in an intensive case management program. Psychiatric Services, 46, 365-371.

Quinlivan, R., \& McWhirter, D. P. (1996). Designing a comprehensive care program for high-cost clients in a managed care environment. Psychiatric Service, 47, 813-815.

Saarento, O., Hakko, H., \& Joukamaa, M. (1998). Repeated use of psychiatric emergency out-patient services among new patients: A 3-year follow-up study. Acta Psychiatrica Scandinavica, 98, 276-282.

Segal, S. P., Akutsu, P. D., \& Watson, M. A. (1998). Factors associated with involuntary return to a psychiatric emergency service within 12 months. Psychiatric Services, 49, 1212-1217.

Silva, N. C., Bassani, D. G., \& Palazzo, L. S. (2009). A case-control study of factors associated with multiple psychiatric readmissions. Psychiatric Services, 60, 786-791.

Snowden, L. R. (2007). Explaining mental health treatment disparities: Ethnic and cultural differences in family involvement. Culture, Medicine and Psychiatry, 31, 389-402.
Sullivan, P. F., Bulik, C. M., Forman, S. D., \& Mezzich, J. E. (1993). Characteristics of repeat users of a psychiatric emergency service. Hospital \& Community Psychiatry, 44, 376-380.

Surber, R. W., Winkler, E. L., Monteleone, M., Havassy, B. E., Goldfinger, S. M., \& Hopkin, J. T. (1987). Characteristics of high users of acute psychiatric inpatient services. Hospital \& Community Psychiatry, 38, 1112-1114.

Surles, R. C., \& McGurrin, M. C. (1987). Increased use of psychiatric emergency services by young chronic mentally ill patient. Hospital \& Community Psychiatry, 38, 401-405.

U.S.Census Bureau, \& SANDAG. (2000). Population by race and Hispanic/Latino origin by Jurisdiction San Diego region. http://www.sandag.org/uploads/publicationid/publicationid_ 469_620.pdf.

Wingerson, D., Russo, J., Ries, R., Dagadakis, C., \& Roy-Byrne, P. (2001). Use of psychiatric emergency services and enrollment status in a public managed mental health care plan. Psychiatric Services, 52, 1494-1501.

Witheridge, T. F., Dincin, J., \& Appleby, L. (1982). Working with the most frequent recidivists: A total team approach to assertive resource management. Psychosocial Rehabilitation Journal, 5, $9-11$.

Young, A. S., Chinman, M. J., Cradock-O'Leary, J. A., Sullivan, G., Murata, D., Mintz, J., et al. (2005). Characteristics of individuals with severe mental illness who use emergency services. Community Mental Health Journal, 41, 159-168. 